\title{
Relaxation mechanisms in magnetic colloids studied by stroboscopic spin-polarized small-angle neutron scattering
}

\author{
A. Wiedenmann,,${ }^{1, *}$ R. Gähler, ${ }^{1}$ C. D. Dewhurst, ${ }^{1}$ U. Keiderling, ${ }^{2}$ S. Prévost,${ }^{3}$ and J. Kohlbrecher ${ }^{4}$ \\ ${ }^{1}$ Institute Laue Langevin, Av. Jules Horowitz, LSS, F38042 Grenoble Cedex, France \\ ${ }^{2}$ Helmholtz Center Berlin, D-14109 Berlin, Germany \\ ${ }^{3}$ Technical University Berlin, D-10623 Berlin, Germany \\ ${ }^{4}$ Laboratory for Neutron Scattering, ETH Zurich \& PSI, CH-5232 Villigen PSI, Switzerland \\ (Received 5 August 2011; revised manuscript received 2 November 2011; published 6 December 2011)
}

\begin{abstract}
Stroboscopic small-angle neutron scattering by using polarized neutrons is presented as a technique for investigations of relaxation phenomena on a nanometer scale. Applying a cyclic perturbation to the sample by an oscillating magnetic field allows the polarization-dependent scattering cross-sections to be extracted as timedependent response. The potential of this special type of "physical" contrast variation technique is demonstrated with three examples of ferrofluids of different particle sizes and viscosities. The dynamics of magnetic moment reorientations turned out to be governed by different time scales. For a temperature-dependent fraction of individual mobile particles, a frequency-dependent phase shift and damping of the intensity modulation have been precisely determined and quantitatively explained using a Debye-like relaxation model. The characteristic relaxation times, $\tau$, in the liquid state of all samples scale with the particle volume and viscosity confirming a Brownian-relaxation mechanism. For the remaining fraction of particles that form locally ordered domains the orientation distribution of the dynamically arrested static moments has been quantified by an order parameter $S_{1}{ }^{\text {arr }}$, which is not accessible with nonpolarized neutrons. In a high viscous Co and a magnetite-based ferrofluid, $S_{1}$ arr corresponds to maximal alignment of the static moments in the liquid state and to a gradual decrease at lower temperatures when the solvent is frozen. In a low viscosity Co-ferrofluid almost no preferred alignment of the arrested particle moments was observed. In the magnetite-based ferrofluid with the highest dipolar attraction energy an additional phase shift has been detected which increases with frequency and magnitude of the magnetic field. This indicates clearly that the set-up of local particle ordering in distorted hexagonal arrangement is delayed with respect to the moment alignment in the time varying magnetic field.
\end{abstract}

DOI: 10.1103/PhysRevB.84.214303

PACS number(s): 75.25.-j, 75.50.Mm, 75.50.Tt, 75.75.-c

\section{INTRODUCTION}

Time-resolved small-angle neutron scattering (SANS) techniques have recently been developed, which allow ordering and relaxation processes of magnetic moments in nanoparticles to be monitored. ${ }^{1-3}$ While ac-susceptibility ${ }^{4}$ and relaxometry techniques ${ }^{5}$ probe the macroscopic sample magnetization, the stroboscopic SANS is sensitive to local fluctuations of the magnetic moment with accessible time much longer than those of Neutron Spin-Echo techniques ${ }^{6,7}$ or Mössbauer spectroscopy ${ }^{8}\left(10^{-6}-10^{-12} \mathrm{~s}\right)$ but complementary to $\mathrm{x}$-ray photon correlation spectroscopy ${ }^{9}$ and forced Rayleigh scattering. ${ }^{10}$ Field-induced ordering phenomena in magnetic colloids have been extensively studied in the past decade, ${ }^{11}$ for example, in surfactant stabilized ferrofluids (denoted as FF) with $\mathrm{Co}^{12}$ and $\mathrm{Fe}_{3} \mathrm{O}_{4}{ }^{13,14}$ particles of low polydispersity. When an external static magnetic field was applied, domains of local hexagonal ordering formed by nanosized magnetic particles have been found to coexist with flexible dipolar chains. ${ }^{12}$

In a first type of stroboscopic SANS experiments, the relaxation of the local ordering toward equilibrium has been studied when the saturation magnetic field was switched off. ${ }^{1}$ Magnetic correlations between Co-nanoparticles were found to decay exponentially with a characteristic time of the order of 1 to $5 \mathrm{~s}$. In a second type of experiment the time-resolved SANS response to a periodic perturbation from a sine-wave modulation of the external magnetic field was analyzed by two techniques ${ }^{2}$ : a direct stroboscopic mode, which uses a continuous neutron beam, and a new pulsed time-resolved technique TISANE, which allows exploitation of a dynamical range extended to submilliseconds similar to that of x-ray photon-correlation spectroscopy. The analysis of time-dependent SANS data as a function of frequency, field, and temperature ${ }^{3}$ has previously allowed us to elucidate the dynamical nature of the locally ordered domains in both FFs, as "living objects" becoming arrested below the freezing temperature of the solvent. The time-dependent intensities have been analyzed in terms of Langevin statistics, including dynamical interparticle structure factors determined by the effective dipole-dipole interaction. Starting from the frozen state, the amount of freely rotating particle moments increases continuously with increasing temperature. The alignment of particle moments along the applied field is governed by the fast Brownian rotation of individual nanoparticles and small aggregates, while the magnetic relaxation of longer dipolar chains and local hexagonal domains is much slower.

Here we focus on an extension of the stroboscopic investigations using polarized neutrons with polarization analysis of the scattered neutron spin (POLARIS). The continuous neutron beam technique was applied, which limits the maximum resoluble frequency to $300 \mathrm{~Hz}$ due to the neutron time-of-flight spread (TOF) resulting from the wavelength spread $\Delta \lambda / \lambda$ $=0.1$. Upon decreasing the temperature the characteristic relaxation time is expected to increase depending on the mechanism and the relative interactions involved. The aim of this study was to demonstrate that (i) the neutron polarization is not perturbed by the oscillating field, and (ii) more detailed 
insight into the dynamical processes of magnetic colloids is obtained when polarized neutrons are used. For this purpose we focus on the same FF samples of different core volumes and viscosities as used in previous studies with nonpolarized neutrons and, as before, ${ }^{3}$ we restrict the analysis to few selected areas in $Q$-space, which are relevant for the set-up of field induced ordering.

\section{SCATTERING CROSS-SECTIONS}

For ferromagnetic single-domain particles embedded in a nonmagnetic matrix the orientation distribution of the magnetic moments in an effective field $\mathbf{B}$ follows Langevin statistics which can be described as

$$
p(\theta, \alpha)=\frac{\alpha}{4 \pi \sinh (\alpha)} \exp (\alpha \cos (\theta)),
$$

where $\theta=\varangle(\mathbf{B}, \mathbf{M})$ is the angle between the direction of the applied magnetic field $\mathbf{B}$ and the direction of the magnetization of the particle $\mathbf{M}$, and $\alpha$ is the Lagrange parameter

$$
\alpha=\frac{V_{c} \cdot m_{0} \cdot B(t)}{k_{B} \cdot T} .
$$

$V_{c}$ and $m_{0}$ are the volume and the saturation magnetization of the ferromagnetic core respectively. The orientation distribution can be expanded in terms of a complete set of orthogonal Legendre polynomials $P_{l}(\cos (\theta))$ as

$$
p(\theta, \alpha)=\sum_{l} \frac{2 l+1}{2} S_{l} P_{l}(\cos (\theta)) .
$$

$S_{l}$ are order parameters which can be calculated from $S_{l}=$ $\int_{0}^{\pi} p(\theta, \alpha) P_{l}(\cos (\theta)) \sin (\theta) d \theta$, and the first of them are given in case of Langevin statistics by

$$
\begin{gathered}
S_{0}=1 \\
S_{1}=L(\alpha)=\operatorname{coth}(\alpha)-\frac{1}{\alpha} \\
S_{2}=1-3 \frac{L(\alpha)}{\alpha} .
\end{gathered}
$$

The first-order parameter $S_{1}$ is equivalent to the magnetization and can have values between -1 and 1 . A value of 1 means perfect alignment along an external field, 0 means fully random orientation, and -1 signifies antiparallel alignment to the external field. The second-order parameter $S_{2}$ can take values between -0.5 and 1 , where a value of 1 means an orientation parallel or antiparallel to the external field, a value of 0 means random orientation, and a value of -0.5 means perpendicular orientation to the external field. The second, and all other even-order parameters, are independent of a $\pi$ rotation of the particle. It can be shown that the SANS pattern only depends on a few order parameters that can be obtained from the orientation distribution. When polarized neutrons are used with the neutron spins aligned antiparallel, denoted by $(+)$, or parallel (-), to a preferred orientation $\mathbf{z} / / \mathbf{H}$, four types of scattering process can be distinguished: two for conserving the neutron spin (spin nonflip scattering: snf) and two with reversal of the spin by the scattering (spin-flip scattering: sf). ${ }^{15-17}$ The four scattering cross-sections $\frac{d \sigma_{i j}}{d \Omega}(Q, \alpha)$ with $i, j=+,-$ are derived for the case where the scattering vector, $\mathbf{Q}$, may form any angle, $\Psi$, with the applied magnetic field, $\mathbf{H}$, lying in the plane of the detector and perpendicular to the incoming neutron beam. The scattering contributions can be written in the decoupling approach as

$$
\begin{aligned}
\frac{d \sigma_{i j}}{d \Omega}(Q, \Psi) & =\left\langle F_{i j}^{2}\right\rangle+\left\langle F_{i j}\right\rangle^{2}[S(Q, \Psi)-1] \\
& =\left\langle F_{i j}\right\rangle^{2} S(Q, \Psi)+\left\langle F_{i j}^{2}\right\rangle-\left\langle F_{i j}\right\rangle^{2} \\
& =A_{i j}(Q) S(Q, \Psi)+B_{i j}(Q) F_{M}^{2}(Q),
\end{aligned}
$$

where $\left\langle F_{i j}^{2}\right\rangle$ and $\left\langle F_{i j}\right\rangle^{2}$ are the averaged squared form factor and the squared average form factors, respectively. ${ }^{19}$ These averages depend differently on the order parameters $S_{1}$ and $S_{2}$ for the scattering processes,,++--+- and -+ , as summarized in Table I. With polarization analysis (POLARIS) the four cross-sections can be extracted individually. Without polarization analysis (SANSPOL) snf and sf scattering are summed up in the detector leading to two different intensities $I_{-}(Q, \Psi)$ and $I_{+}(Q, \Psi)$, the average of which corresponds to the intensity of nonpolarized SANS. The first term $A_{i j}(Q)$ in Eq. (5) is a contribution subject to nuclear and/or magnetic interparticle correlations described by $S(Q, \Psi)$, while the second term $B_{i j}(Q)$ results solely from nonperfect alignment of the magnetic moments of individual particles. In the case of a static measurement at a given magnetic field at least four independent SANS contrasts are needed to determine nuclear $\left(F_{N}(Q)\right)$ and magnetic particle form factors $\left(F_{M}(Q)\right)$, the structure factor $S(Q, \Psi)$, and the argument $\alpha$ in Eq. (2). Some of these values can be obtained from the analysis of the full $Q$-dependence of the SANS data, e.g., the argument $\alpha$ can be calculated according to Eq. (2) once the particle size and the magnetic moment $m_{0}$ are known. When POLARIS data are used the static nuclear and magnetic nanostructure of the material can be fully determined from simultaneous fits of independent-scattering intensities $I_{ \pm \pm ⿱ 土 土}(Q)$ for sf and nonspin flip (nsf). When additional assumptions such as the isotropic character of $S(Q, \Psi)=S(Q)=1$ for diluted samples are justified, SANSPOL or SANS data, respectively, are sufficient to meet the goal.

An oscillating magnetic field can be described by

$$
B(t)=B_{0} \cdot \cos \left(2 \cdot \pi \cdot v_{S} \cdot\left(t+t_{\mathrm{tof}}\right)+\phi_{0}\right)+B_{s t},
$$

where $B_{0}$ and $B_{s t}$ are the amplitudes of the ac field and of the superimposed static field, respectively, and $\phi_{0}$ is an experimental phase shift resulting from electronic components. A time dependence enters Eq. (5) via the argument $\alpha(t)$ of the Langevin function $L(\alpha(t))$. Equation (5) has to be convoluted with the time resolution of the instrument resulting from the triangular wavelength distribution $p_{\lambda}(\lambda, \Delta \lambda)$. The time-dependent SANS intensity is given by

$$
\begin{aligned}
I_{i j}(Q, \Psi, t)= & f_{e} \int\left[A_{i j} S(Q, \Psi, t)+B_{i j} F_{M}^{2}(Q)\right] \\
& \times p_{\lambda}(\lambda, \Delta \lambda) d \lambda+\left(1-f_{e}\right) U\left(Q, \Psi, v_{S}\right) .
\end{aligned}
$$

The first term is the instantaneous response of the fraction of superparamagnetic moments $f_{e}$ that can follow the oscillating field without any delay, and the second term is a timeindependent scattering contribution resulting from the static particles. 
TABLE I. Weighting functions for the scattering contributions of interparticle correlations $A_{i j}(Q) S(Q, \Psi)$ and of moment misalignment $B_{i j}(Q) F_{M}^{2}(Q)$, respectively, with the abbreviations $a=\left(1-S_{2}\right) / 3$ and $b=S_{1}^{2}-S_{2}$. In the present case of superparmagnetic single domain particles $a=\frac{1-S_{2}}{3}=\frac{L(\alpha)}{\alpha}$ and $b=S_{1}^{2}-S_{2}=\left[L^{2}(\alpha)-1+3 \frac{L(\alpha)}{\alpha}\right]$, where $L(\alpha)$ is the Langevin function. In this case the weighting functions are determined by the values compiled in Ref. 17. $A_{i j}(Q)$ converges for the limiting case of zero field to $A_{i j}(Q) \underset{B \rightarrow 0}{\longrightarrow} F_{N}^{2}$. $F_{N}$ and $F_{M}$ are the nuclear $(N)$ and magnetic $(M)$ form factors of the particles defined by $F_{N, M}(Q, R)=\int_{0}^{R} d r \cdot \Delta \eta_{N, M}(r) \cdot 2 \pi r^{2} \frac{\sin (Q \cdot r)}{Q r}$ with the corresponding radial symmetric scattering length density profiles $\Delta \eta_{N, M}(r)=\eta_{N, M}(r)-\eta_{\text {matrix }}$. For nonspherical symmetric particles the whole formalism only holds if the magnetization direction of the single domain particles and the particle shape are independent.

\begin{tabular}{|c|c|c|c|}
\hline Process & $A_{i j}=\left\langle F_{i j}\right\rangle^{2}$ & $B_{i j}=\frac{\left\langle F_{i j}^{2}\right\rangle-\left\langle F_{i j}\right\rangle^{2}}{F_{M}^{2}}$ & $B_{i j}(B \rightarrow 0)$ \\
\hline \multicolumn{4}{|l|}{ POLARIS } \\
\hline$I_{--}(Q)$ & $\left(F_{M} S_{1} \sin ^{2} \Psi+F_{N}\right)^{2}$ & $a \sin ^{2} \Psi-b \sin ^{4} \Psi$ & $\frac{1}{3} \sin ^{2} \Psi$ \\
\hline$I_{++}(Q)$ & $\left(F_{M} S_{1} \sin ^{2} \Psi-F_{N}\right)^{2}$ & $a \sin ^{2} \Psi-b \sin ^{4} \Psi$ & $\frac{1}{3} \sin ^{2} \Psi$ \\
\hline$I_{ \pm \mp}(Q)$ & $\left(F_{M} S_{1} \sin \Psi \cos \Psi\right)^{2}$ & $\begin{array}{l}a\left(2-\sin ^{2} \Psi\right) \\
-b \sin ^{2} \Psi \cos ^{2} \Psi\end{array}$ & $\frac{1}{3}\left(2-\sin ^{2} \Psi\right)$ \\
\hline $\begin{array}{l}I_{--}(Q)-I_{++}(Q) \\
\text { SANSPOL }\end{array}$ & \multicolumn{2}{|c|}{ SANSPOL } & 0 \\
\hline$I_{-}(Q)=I_{--}(Q)+I_{-+}(Q)$ & {$\left[F_{M}^{2} S_{1}^{2}+2 F_{N} F_{M} S_{1}\right] \sin ^{2} \Psi+F_{N}^{2}$} & $2 a-b \sin ^{2} \Psi$ & $2 / 3$ \\
\hline$I_{+}(Q)=I_{++}(Q)+I_{+-}(Q)$ & {$\left[F_{M}^{2} S_{1}^{2}-2 F_{N} F_{M} S_{1}\right] \sin ^{2} \Psi+F_{N}^{2}$} & $2 a-b \sin ^{2} \Psi$ & $2 / 3$ \\
\hline$I_{-}(Q)-I_{+}(Q)$ & $4 F_{N} F_{M} S_{1} \sin ^{2} \Psi$ & 0 & 0 \\
\hline \multicolumn{4}{|l|}{ SANS } \\
\hline$I_{\text {unp }}(Q)=\frac{1}{2}\left(I_{-}(Q)+I_{+}(Q)\right)$ & $F_{M}^{2} S_{1}^{2} \sin (\Psi)+F_{N}^{2}$ & $2 a-b \sin ^{2} \Psi$ & $2 / 3$ \\
\hline
\end{tabular}

In real systems at least two deviations from this idealized behavior might appear:

(i) In an intermediate time range where particle moments are not fully static but follow the applied field within some delay we expect a phase shift $\phi_{1}$ between the inducing ac field and the response. In the simplest case the argument $\alpha$ in Eq. (2) is then given by

$$
\begin{gathered}
\alpha(t)=D\left(\phi_{1}\right) V_{c} m_{0} B\left(t, \phi_{1}\right) / k_{B} T \\
B\left(t, \phi_{1}\right)=B_{0} \cdot \sin \left(2 \pi v_{S}\left(t+t_{\mathrm{tof}}\right)+\phi_{0}-\phi_{1}\right)+B_{s t},
\end{gathered}
$$

and $D\left(\phi_{1}\right)$ is a damping factor.

(ii) The dynamical structure factor $S(Q, \Psi, t)$ in Eq. (5) might depend on the applied magnetic field. In fact in concentrated FFs $S(Q, \Psi, t)$ has been found to scale according to Ref. 16,

$$
S(Q, \Psi, t)=1+[S(Q, \Psi)-1] L^{2}(\alpha(t)) .
$$

Depending on the strength of the applied magnetic field, dipolar interactions give rise to different types of locally

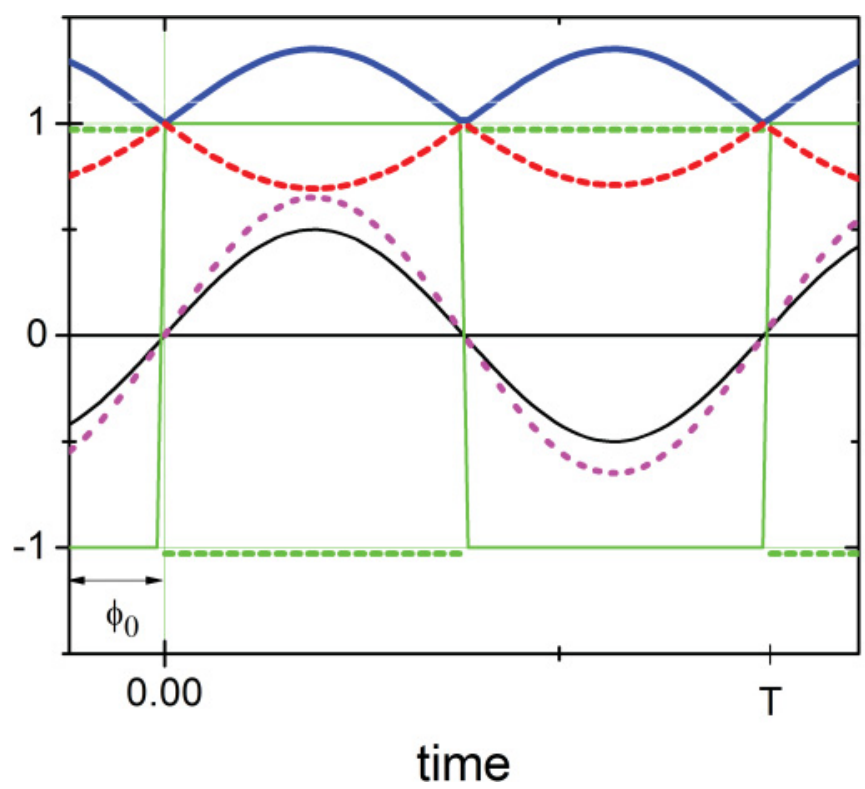

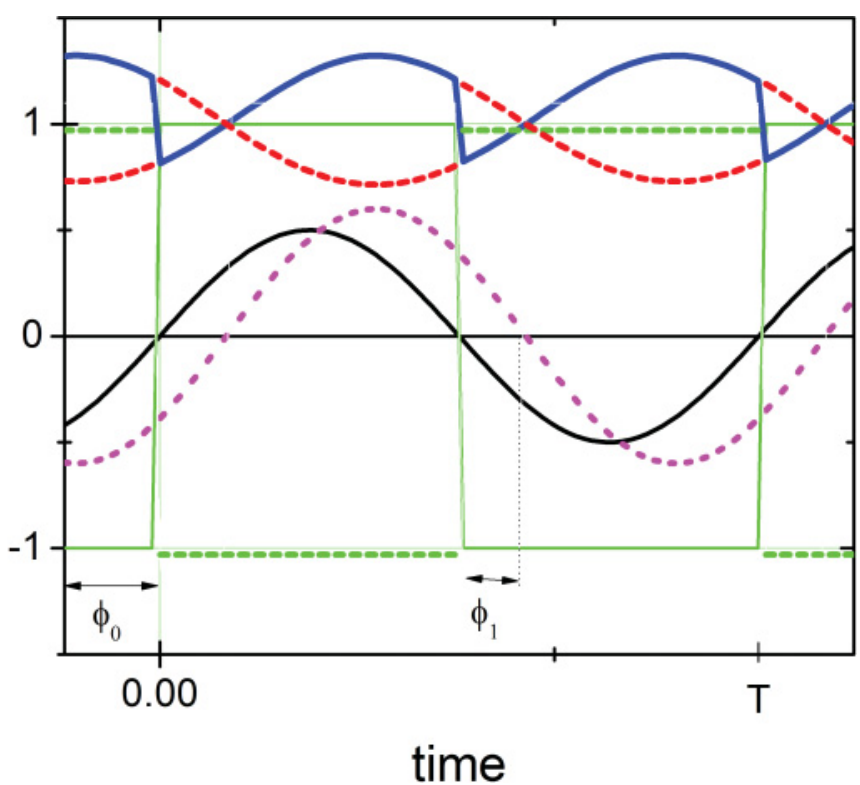

FIG. 1. (Color online) Schematic representation of the time dependency of magnetic field $B(t)$ (black/solid thin line), the particle moment $M(t)$ (pink/light gray dashed line), the sign of the neutron spins (-) (green/gray full line) and (+) (green/gray dashed line), and the SANSPOL intensities $I(-)_{\mathrm{Q} \perp \mathrm{B}}$ (blue/medium gray solid line) and $I(+)_{\mathrm{Q} \perp \mathrm{B}}($ red/dark gray dashed line). Left side: without delay of $M(t)$ with respect to $B(t)$; right side: delayed response. $\phi_{0}$ is an instrumental phase. 
ordered domains such as chains, bands, or hexagonal particle arrangements. In an oscillating magnetic field the onset of interparticle correlations might be delayed with respect to the reorientation of the magnetic particle moment, i.e., a different phase shift $\phi_{2}$ may occur between $L(t)$ and $S(t)$.

Polarized SANS with or without polarization analysis has successfully been used for precise characterization of magnetic nanostructures. ${ }^{17,18}$ When a static magnetic field is applied one takes advantage of different contrasts, i.e., different weighting functions $A_{i j}$ and $B_{i j}$ in Table I. The first goal of the present study was to demonstrate that polarized neutrons can be used in a stroboscopic technique for the purpose of a "physical" contrast variation. In the presence of a guide field of $1 \mathrm{mT}$ the Larmor frequency of neutron spin is $v_{L}=\gamma_{L} H=29.165 \mathrm{kHz}$, i.e., four orders of magnitude faster than the frequency of the applied oscillating field. Hence we expect that the neutron spin will follow adiabatically the oscillating field and depolarization of the beam is unlikely. The time dependence of the field $B(t)$, the magnetization $M(t)$, and the expected POLARIS cross-sections are schematically shown in Fig. 1. In the present frequency range the neutron spin (green line) always follows the oscillating field $B(t)$ (black thin line). When there is no delay of the magnetization $M(t)$ (pink line) with respect to $B(t)$ (Fig. 1, left side), the cross-section $I_{--}$(full blue line) is observed for the whole time period for ( - ) neutrons (full green line) as well as the $I_{++}$ for $(+)$ neutrons. Any delay in the reorientation of magnetic particle moments or of the local ordering (Fig. 1, right side) gives rise to a phase shift of $M(t)$ by $\phi_{1}$ or of $S(Q, t)$ by $\phi_{2}$, respectively. In a certain time range the neutron spin and particle moment are no longer in phase, and the time-dependent nsf scattering intensity will jump from $(++)$ to $(--)$ or vice versa. Similarly, when the moments are static and aligned we expect a change from a time-independent intensity $(++)$ to $(--)$ at half the time period when $B(t)$ changes its sign. Similarly, the sf scattering is expected to jump from $(+-)$ to $(-+)$. However, since in the present case of a nonchiral system $I_{+-}$and $I_{-+}$are identical, the sf contribution is not sensitive to a phase shift. For SANSPOL where sf and nsf are mixed we still expect a significant change of the $I_{-}$and $I_{+}$intensities when such a phase shift appears. The field dependence of the interparticle interference term $S(Q, t)$ and the corresponding phase shift $\phi_{2}$ SANSPOL difference intensity $I_{-}-I_{+}$should be the most sensitive observable since the contribution from spin-misalignment $B_{i j}$ is zero (see Table I).
For unpolarized neutrons, a phase shift results in only slightly different absolute values of the contrast, while for polarized neutrons a discontinuity in the contrast is expected (Fig. 1). Under adiabatic conditions the stroboscopic technique with polarized neutrons is expected to be extremely sensitive to the delayed response, i.e., to the analysis of relaxation times.

\section{EXPERIMENTAL}

SANSPOL measurements have been performed on the instrument D22 at ILL equipped with a polarizing supermirror and a RF gradient flipper using a wavelength of $\lambda=6 \AA$, $\Delta \lambda / \lambda=0.1$, and a distance of $4 \mathrm{~m}$ between sample and detector. We used the same FF samples from previous studies with nonpolarized neutrons, namely a concentrated highly viscous Co-FF dispersed in L9 oil "MFT3N,"3 magnetiteFF dispersed in decalin ${ }^{14}$ and Co-FF "77-4" in deuterated toluene. The relevant structural and magnetic parameters of the samples are reported in Table II.

Polarization analysis on "MFT3N"Co-FF was performed with a ${ }^{3} \mathrm{He}$ filter incorporated in the "magic box," 19 a uniform magnetic field cavity which includes a nuclear polarization flipping device. The life time of the filter cell with an initial ${ }^{3}$ He polarization of $75 \%$ was of the order of 200 hours such that the polarizing efficiency was essentially constant during the POLARIS measurements. We performed continuous stroboscopic measurements at frequencies between $v_{\mathrm{s}}=100 \mathrm{~Hz}$ and $300 \mathrm{~Hz}$ for which the relative time resolution varied according to $\Delta t / T_{\mathrm{s}}=\Delta \lambda L_{1} v_{\mathrm{s}} / 3956$ between $6 \%$ and $13 \%$, respectively, resulting from spread of TOF $\Delta t$ (in seconds) on the flight path $L_{1}=4 \mathrm{~m}$ for the distribution of wavelengths $\Delta \lambda$ (in $\AA$ ). An oscillating magnetic field in horizontal direction perpendicular to the neutron beam up to amplitudes of $B_{0}=20 \mathrm{mT}$ and additional static fields up to $B_{\mathrm{st}}=20 \mathrm{mT}$ were produced by a magnetic coil with ferrite core powered by a frequency generator and ac-power amplifier. TOF mode data acquisition was synchronized with the oscillating field and 2-dimensional SANS data were regrouped into 100 time channels. The samples sealed in quartz containers were cooled down in a closed cycle refrigerator to $20 \mathrm{~K}$. Standard cell-by-cell data corrections and regrouping of time-dependent data have been performed by the software package BerSANS. ${ }^{20}$ Instead of analyzing the full $3 \mathrm{D}$ intensity pattern $I(Q, \psi, t)$, we first integrated the intensity over a restricted $Q$-range region of

TABLE II. Structural and magnetic parameters of the different FF samples.

\begin{tabular}{lccc}
\hline \hline Sample & Co-FF $(\mathrm{MFT} 3)$ & $\mathrm{Fe}_{3} \mathrm{O}_{4}$-FF & Co-FF 77-4 \\
\hline Surfactant shell thickness $d_{\mathrm{s}}(\mathrm{nm})$ & $1.9 \pm 0.2$ & $2.1 \pm 0.2$ & $8.2 \pm 0.2$ \\
Core-radius $R_{c}(\mathrm{~nm})$ & $4.4 \pm 0.2$ & 10 & $1.9 \pm 0.2$ \\
Poly-dispersity $(\%)$ & 15 & 15.5 & $2.7 \pm 0.2$ \\
Magnetic particle moment $\mu\left(10^{19} \mathrm{~A} \cdot \mathrm{m}^{2}\right)$ & 5.3 & -9 & 15 \\
Dipole-dipole attraction $E_{d d}(\max )\left(\mathrm{k}_{\mathrm{B}} \cdot \mathrm{T}\right)$ & -4 & -15.2 & -0.55 \\
Interaction $E_{B}$ with $40 \mathrm{mT}$ at $300 \mathrm{~K}\left(k_{\mathrm{B}} \cdot T\right)$ & -5.2 & 5 & -1.2 \\
Contrast ratio $\Delta \eta_{\text {nuc }} / \Delta \eta_{\text {mag }}$ & 0.29 & decalin & -1.1 \\
Solvent/matrix & $\mathrm{L}$ & $2.510^{-3}$ & $\mathrm{D}-\mathrm{toluene}$ \\
Viscosity solvent $(\mathrm{Pa} \cdot \mathrm{s})$ & 0.2 & $0.610^{-3}$ \\
\hline \hline
\end{tabular}


interest (ROI) $(\Delta Q, \Delta \psi)$, which have been chosen to cover the interparticle correlation peaks known from previous SANS investigations. $^{12-14}$ The time dependence of different ROIs has been analyzed in terms of Eq. (7). This simplification implies that polydispersity is treated by assuming an average particle size with an Langevin parameter $\alpha(t)$ (Eq. (8)) and with fixed-averaged values of $A_{i j}, B_{i j}, F_{M}(Q)$, and $S(Q, \psi)$ in Eqs. (7) and (10). In the present case of small polydispersity this is justified since the size distribution does not change when the field is applied.

For POLARIS the two nsf cross-sections $d \sigma / \mathrm{d} \Omega(++)$, $d \sigma / \mathrm{d} \Omega(-)$ as well as the spin-flip cross-sections $d \sigma / \mathrm{d} \Omega(+-)$ and $d \sigma / \mathrm{d} \Omega(-+)$ have been extracted from the measured intensities by using the efficiencies of the neutron polarizer $(\mathrm{Pn}=0.92)$ and the spin-flippers $(\varepsilon=0.99)$ and of the (nearly time independent) analyzer efficiency $(\mathrm{A}=0.9)$ of the ${ }^{3} \mathrm{He}$ filter according to the procedure described in Ref. 20. All scattering contributions are described in Table I and
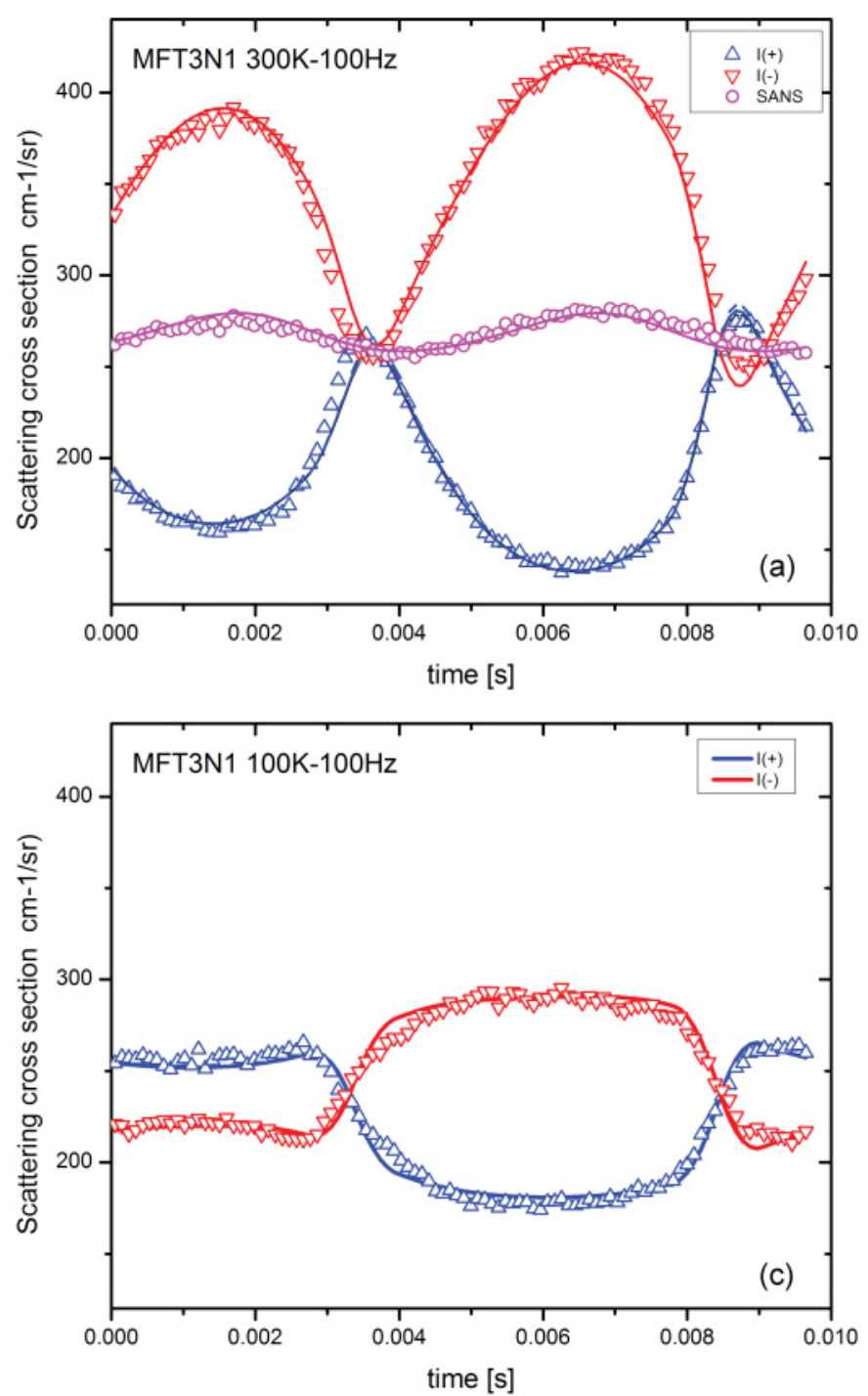

Fig. 1 for POLARIS, SANSPOL, and SANS and have been implemented in the SASFIT software package ${ }^{21}$ as model functions, including phase shifts resulting from experimental set-up $\left(\phi_{0}\right)$ or from delayed response of the magnetization $\left(\phi_{1}\right)$ or the structure factors $\left(\phi_{2}\right)$. The different time-dependent ROI have been adjusted simultaneously, allowing the use of constrains between global parameters. The reversibility of the reordering has been checked by comparing the stroboscopic SANSPOL and POLARIS data for the same ROI with those measured in the same coil device with a static magnetic field of the same amplitude.

\section{RESULTS AND DISCUSSION}

\section{A. MFT3N-highly viscous concentrated Co-FF}

SANSPOL measurements have been performed on highly viscous concentrated Co-FF subject to an oscillating field of $\mathrm{B}=20 \mathrm{mT}$ at 100,200 , and $300 \mathrm{~Hz}$ in the temperature range
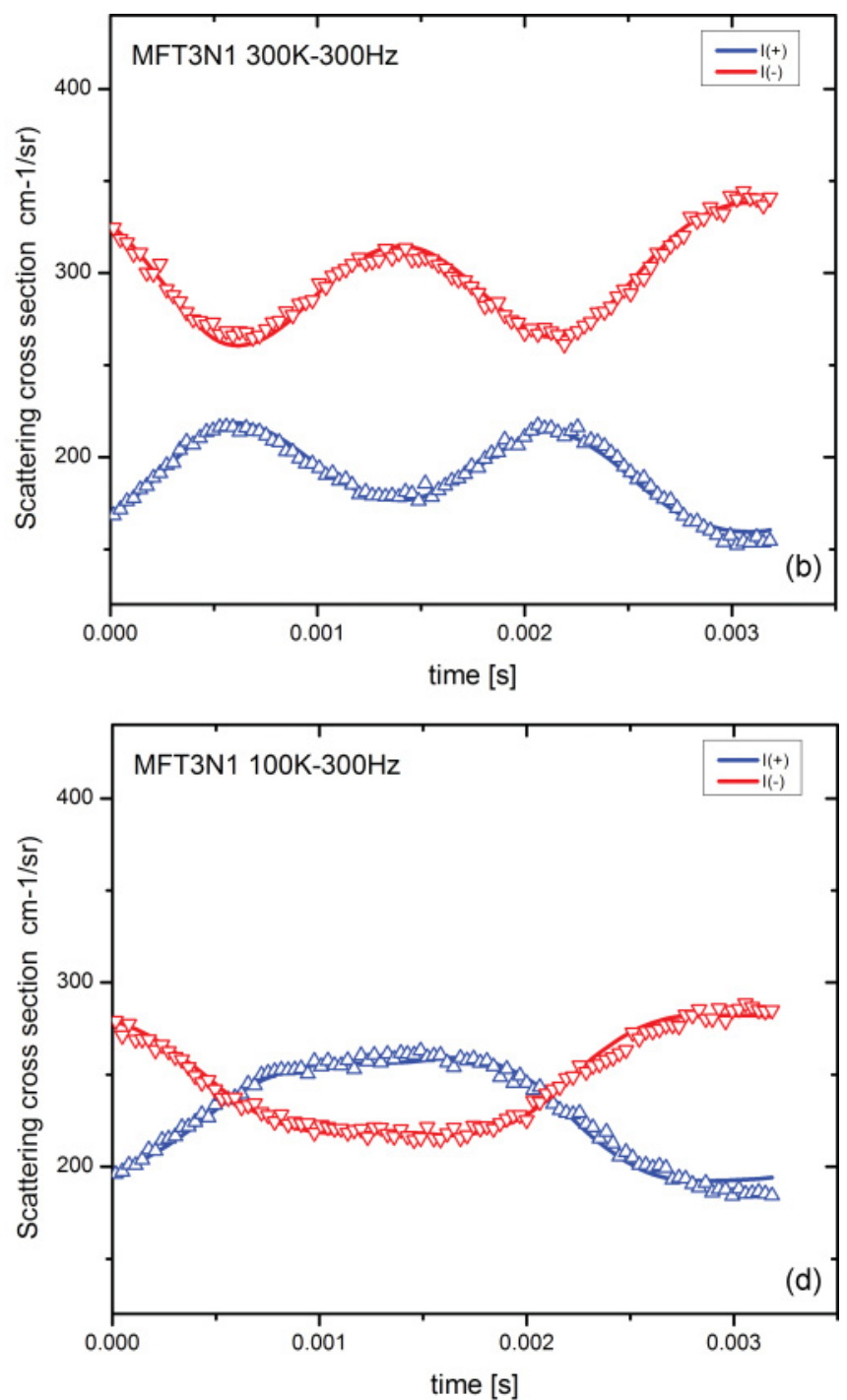

FIG. 2. (Color online) Time dependence of SANSPOL intensities $I_{-}^{\mathbf{Q} \perp \mathbf{B}}$ (red/down triangles) and $I_{+}^{\mathbf{Q} \perp \mathbf{B}}$ (blue/up triangles) at $Q=0.4 \mathrm{~nm}{ }^{-1}$ perpendicular to the applied field measured at $300 \mathrm{~K}$ (a), (b) and $100 \mathrm{~K}(\mathrm{c})$, (d) at frequencies of $100 \mathrm{~Hz}$ (a), (c) and $300 \mathrm{~Hz}$ (b), (d). The solid lines represent a simultaneous model fit according to Eq. (7). The SANS intensity $I(+)+I(-) / 2$ (magenta/circles) in (a) shows much less pronounced contrast. 
between $300 \mathrm{~K}$ and $40 \mathrm{~K}$, crossing the freezing temperature $T_{\mathrm{f}}=119 \mathrm{~K} .{ }^{11}$ In addition, at each temperature measurements have been performed in a static field of the same amplitude. In the following we discuss the time dependence of the SANSPOL intensities $I_{-}^{\mathbf{Q} \perp \mathbf{B}}$ and $I_{+}^{\mathbf{Q} \perp \mathbf{B}}$ (Fig. 2) in a ROI at $Q=0.4 \pm 0.04 \mathrm{~nm}^{-1}$ in the direction perpendicular to the horizontal magnetic field, which corresponds to the interparticle correlation peak, as discussed previously. ${ }^{12}$ The polarized SANS data show a pronounced asymmetry, which increases with decreasing temperature and appears to suppress the oscillation in the first half period while the intensity $I(+)+I(-) / 2$ corresponding to the nonpolarized SANS data in the same ROI at $90^{\circ}$ [Fig. 2(a)] shows a much less pronounced contrast. This behavior supports the interpretation of our previous SANS data ${ }^{2,3}$ based on the superposition of two contributions, a fraction $f$ of particle moments that follows the ac field and the remaining part $(1-f)$ with static moments.
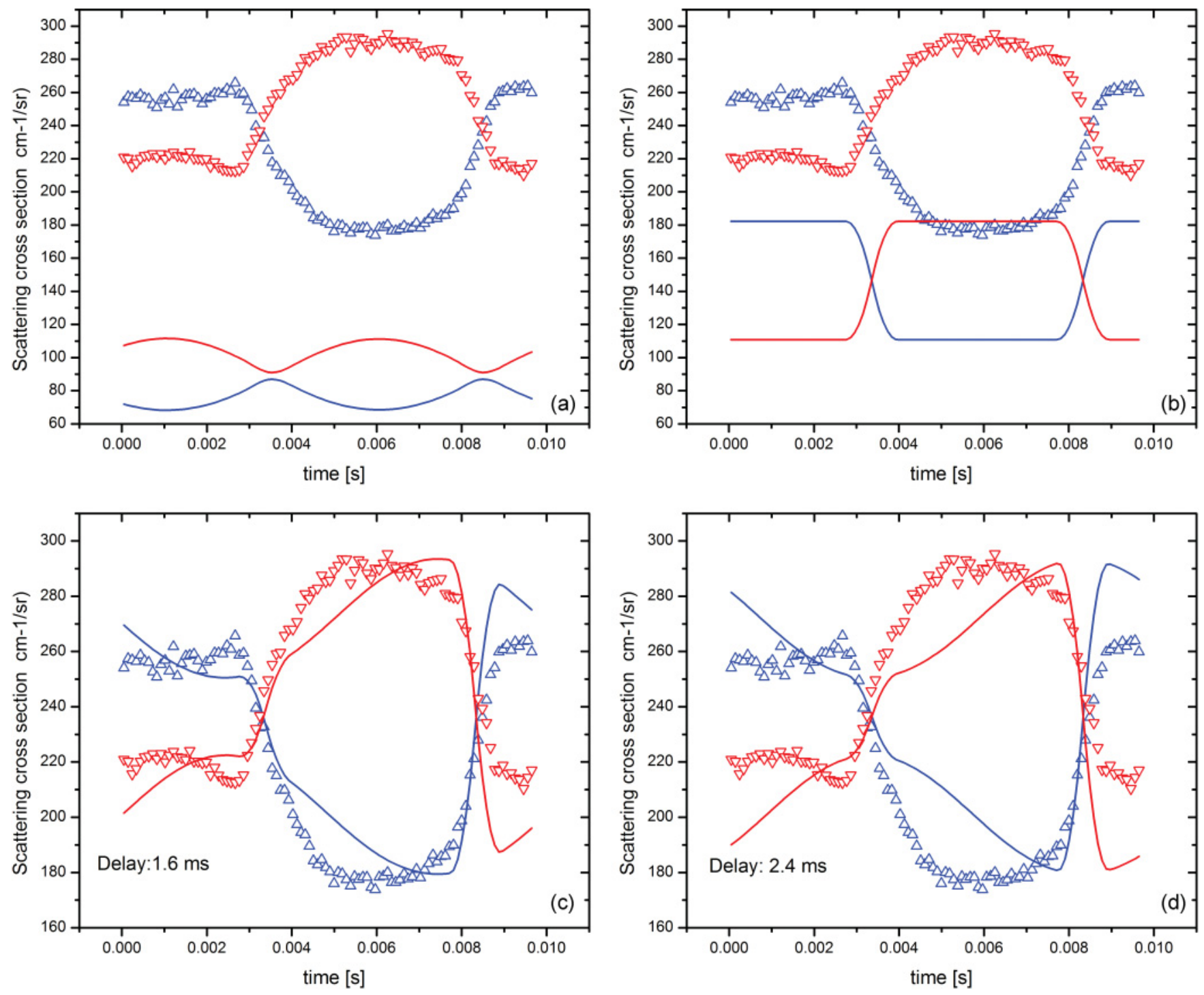

FIG. 3. (Color online) SANSPOL data $I_{-}^{\mathbf{Q} \perp \mathbf{B}}$ (red/down triangles) and $I_{+}^{\mathbf{Q} \perp \mathbf{B}}$ (blue/up triangles) of MFT3N measured at $100 \mathrm{~K}$, $100 \mathrm{~Hz}$. Solid lines represent the contributions from (a) mobile and (b) arrested moments. The effect of a delayed response of the mobile fraction is illustrated in the solid lines of (c) and (d) where the response is calculated for a delay of (c) $1.5 \mathrm{~ms}$ and (d) $2.4 \mathrm{~ms}$, respectively. 

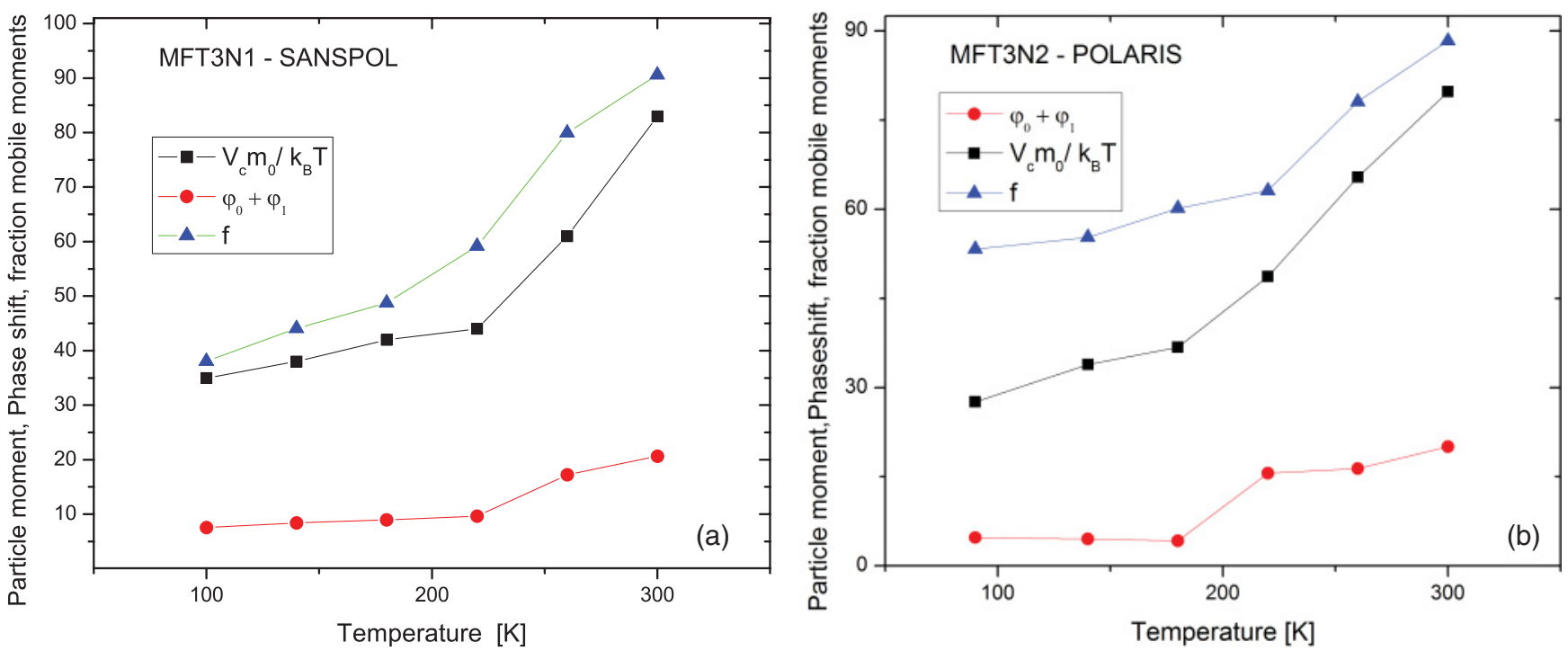

FIG. 4. (Color online) Model parameters volume fraction $f$ (triangles), $\mu / k_{\mathrm{B}} T$ (squares), and phases $\left(\phi_{0}+\phi_{1}\right)$ (circles) for MFT3N from simultaneous fit of SANSPOL (left) and POLARIS data (right).

The full data analysis included a simultaneous fit of $I_{-}^{\mathbf{Q} \perp \mathbf{B}}, I_{+}^{\mathbf{Q} \perp \mathbf{B}}$, and $I_{-}^{\mathbf{Q} \| \mathbf{B}}=I_{+}^{\mathbf{Q} \| \mathbf{B}}$ by using the contrast variation potential of the weighting functions $A_{ \pm}(Q)$ and $B_{ \pm}(Q)$ from Table I. Structural parameters have been constrained as common global model fit parameters for all contributions. The temperature dependence of the parameters is shown in Fig. 4. Stroboscopic measurements were also performed on the same sample MFT3N using a ${ }^{3} \mathrm{He}$ filter for analyzing the polarization of scattered neutrons. The POLARIS transmission values of the sample measured in the oscillating and static field were identical, which indicates that in this frequency range the neutron spin follows the applied field adiabatically with no beam depolarization.

The 2D intensity plots (Fig. 5) from the static measurement at $20 \mathrm{mT}$ show the characteristic angle dependence expected from Table I, i.e., a maximum sf scattering in the direction $\Psi=45^{\circ}$.

All time-dependent POLARIS intensities have therefore been analyzed in the same ROI at $Q=0.4 \pm 0.04 \mathrm{~nm}^{-1}$ but at an angle of $45^{\circ}$. The same previous model was used to analyze nsf and sf data in simultaneous fits giving an excellent agreement with the measured data (solid lines in Fig. 6). Since sf scattering is purely of magnetic origin the observed modulated sf intensity clearly confirms the presence of magnetic correlation in the concentrated FF.

The temperature dependence of the parameter sets obtained from POLARIS and SANSPOL shown in Fig. 4 are remarkably similar. The dominating effect is the decrease of the volume fraction $f$ of moments, which can keep up with the ac-field when cooled below the freezing temperature. The phase parameter changes by about $\Delta \psi \cong 13^{\circ}$, indicating an increasing delay of the response, i.e., a slowing down of the relaxation process. When $\mu / \mathrm{k}_{\mathrm{B}} T$ is adjusted as a free-fit parameter for all contributions (Fig. 4), a strong decrease of the particle moment $\mu=V_{\mathrm{c}} \mu_{0}$ is observed with decreasing temperature. This in turn implies that at low temperatures only the smallest particles can still follow the oscillating magnetic field, and that the static moments must be more and more randomly distributed.

In order to reconcile this phenomenological description in terms of $\phi$ and $f$ with the physics of the processes, we describe the magnetization dynamics in this magnetic colloid by a Debye-like model extended to oscillating fields. At
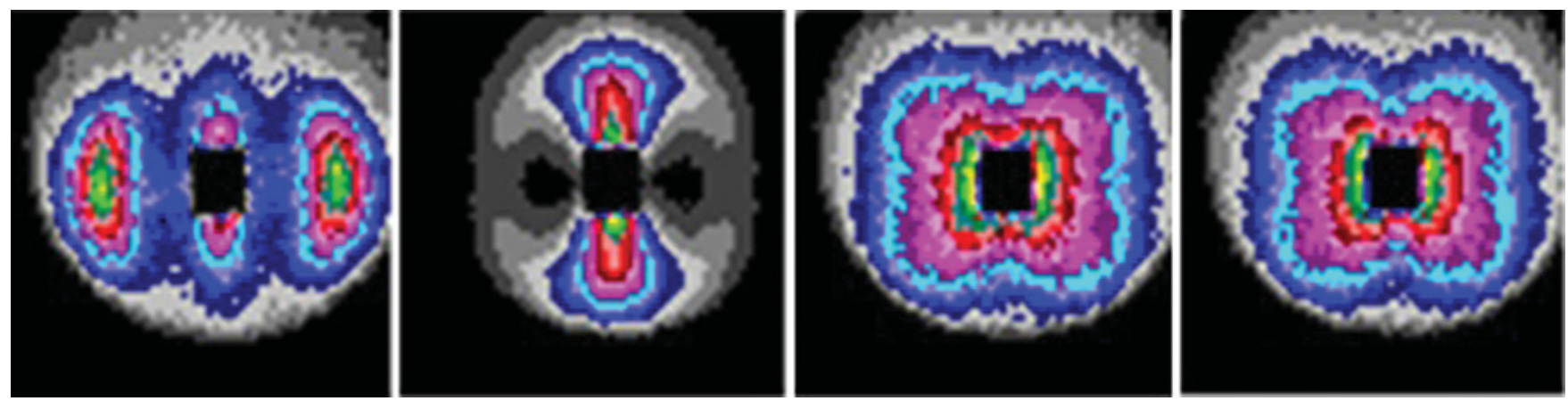

FIG. 5. (Color online) Scattering cross-sections of Co-FF (MFT3N) in L9 extracted from SANSPOL measurements in a horizontal static magnetic field of $20 \mathrm{mT}$ at $300 \mathrm{~K}$, from left to right: $d \sigma_{++} / d \Omega, d \sigma_{--} / d \Omega, d \sigma_{-+} / d \Omega, d \sigma_{+_{-}} / d \Omega$. (The outer part of the detector was shadowed by He filter and masked in the data treatment.) 
any time, $t$, the actual magnetization $M(t)$ is assumed to decay exponentially $M(t)=\langle M\rangle \exp (-t / \tau)$ within a characteristic time, $\tau$, toward the equilibrium value $\langle M\rangle=$ $M_{\infty} L\left(\alpha^{\prime}(t)\right)$ the relaxation rate $\frac{d M}{d t}$ is defined by the differential equation

$$
\frac{d M}{d t}=-\frac{1}{\tau}\left[M(t)-M_{\infty} L\left(\alpha^{\prime}(t)\right)\right]
$$

where $M(t=0)=M_{0}$, and $\alpha^{\prime}(t)$ is the time-dependent Langevin parameter given by

$$
\alpha^{\prime}(t)=\left[B_{0} \cos \left(\omega\left(t-t_{\text {tof }}\right)+\phi_{0}\right)+B_{s t}\right] \mu / k_{B} T .
$$

For small values of $\alpha$ the Langevin function can be approximated by $L(\alpha) \rightarrow \alpha / 3$, and Eq. (11) has an analytical solution given by

$$
\begin{aligned}
M(t)=\exp & \left(-\frac{t}{\tau}\right)\left[M_{0}-M_{\infty} \frac{\mu}{k_{B} T}\left\{\frac{B_{0}}{3} \frac{\cos \left(\phi_{0}-\omega t_{\mathrm{tof}}\right)+\omega \tau \sin \left(\phi_{0}-\omega t_{\mathrm{tof}}\right)}{1+\omega^{2} \tau^{2}}+\frac{B_{s t}}{3}\right\}\right] \\
& +M_{\infty} \frac{\mu}{k_{B} T}\left\{\frac{B_{0}}{3} \frac{\cos \left(\phi_{0}+\omega\left(t-t_{\mathrm{tof}}\right)\right)+\omega \tau \sin \left(\phi_{0}+\omega\left(t-t_{\mathrm{tof}}\right)\right)}{1+\omega^{2} \tau^{2}}+\frac{B_{s t}}{3}\right\} .
\end{aligned}
$$

After the fast exponential decay of the first term in brackets, the oscillating behavior for $t \gg \tau$ can be rewritten as

$$
M(t)=M_{\infty} \frac{\alpha(t)}{3},
$$

where

$$
\begin{aligned}
\alpha(t)= & M_{\infty} \frac{\mu}{k_{B} T}\left\{B _ { 0 } \frac { 1 } { \sqrt { 1 + \omega ^ { 2 } \tau ^ { 2 } } } \operatorname { c o s } \left(\phi_{0}+\omega\left(t-t_{\mathrm{tof}}\right)\right.\right. \\
& \left.\left.-\frac{\pi}{2}+\arcsin \left(\frac{1}{\sqrt{1+\omega^{2} \tau^{2}}}\right)\right)+B_{s t}\right\} .
\end{aligned}
$$

When the magnetic moments follow the external magnetic field with some delay, the oscillating SANS signal can be described by simply introducing a time-dependent value for the Langevin parameter $\alpha(t)$ in Eq. (11) with an additional phase $\phi_{1}$ between $\alpha(t)$ and $B$, and a damping factor $D_{\alpha}$, which

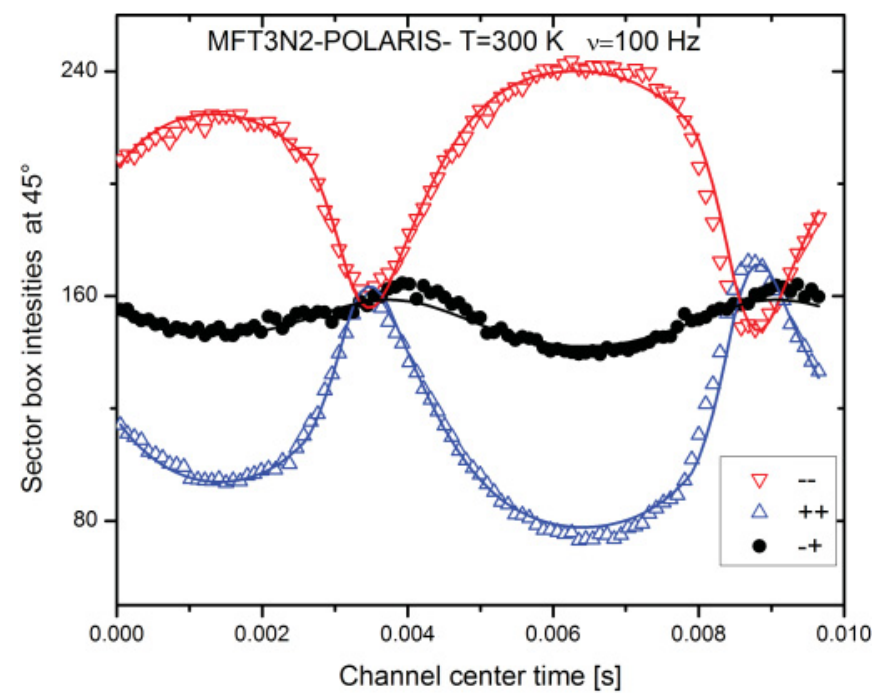

FIG. 6. (Color online) Stroboscopic POLARIS cross-sections at $\psi=45^{\circ} d \sigma / d \Omega(-)$ (red down triangles), ++ (blue up triangles), and $(+-)=(-+)$ (black circles) of MFT3N at $100 \mathrm{~Hz}$ and $300 \mathrm{~K}$, together with the model fit (solid lines) resulting in a set of parameters as shown in Fig. 4(b). are now related to the characteristic time $\tau$ and the frequency $\omega$ by

$$
\begin{gathered}
D_{\alpha}(\omega, \tau)=\frac{1}{\sqrt{1+\omega^{2} \tau^{2}}} \\
\phi_{1}(\omega, \tau)=\arcsin \left(\frac{1}{\sqrt{1+\omega^{2} \tau^{2}}}\right)-\frac{\pi}{2} .
\end{gathered}
$$

In a slowly oscillating field where $\omega \ll 1 / \tau$ the parameter $D_{\alpha} \rightarrow 1$ and $\phi_{1} \rightarrow 0$, i.e., Eq. (6) is valid. When the oscillation period $(2 \pi v)^{-1}$ approaches the characteristic time $\tau$ the damping factor becomes $D_{\alpha}(\omega=1 / \tau, \tau)=0.7$ and the phase shift $\phi_{1}(\omega=1 / \tau, \tau)=-\frac{\pi}{4}$. For very fast oscillating fields where $\omega \gg 1 / \tau \omega \gg 1 / \tau$, one finds $D_{\alpha} \rightarrow 0$ and $\phi_{1} \rightarrow-\frac{\pi}{2}$. In this case where the moments are static the first term in Eq. (11d) is zero, i.e., $L(t)=0$, and the scattering intensity is expected to be independent of time and neutron polarization. However, if there is a static field $B_{\text {st }}$ then $L(t)$ is constant but nonzero, i.e., the time-independent scattering contribution of arrested particles should depend on the polarization. This feature can be used to define an order parameter

$$
S_{1}^{\mathrm{arr}}=L\left(B_{s t} \mu / k_{B} T\right),
$$

which represents the average alignment of the arrested particle moments in a preferred direction along the static (guide) field.

We have applied this general model by using a constant particle moment $\mu=m_{0} V_{c}$ corresponding to the average core volume $V_{c}={ }^{4} / 3^{\pi} R_{c}^{3}$ and the temperature $T$ of the sample for calculating the argument $\alpha=B(t) \mu / k_{B} T$, i.e., we assume superparamagnetic behavior. We then treat the fraction of oscillating particles with the previously defined Debye-like relaxation model with a characteristic time $\tau$ to be determined. This implies a damping of the oscillations to a constant intensity and an increasing phase shift when the relaxation is slowed down.

The fraction of static particles is taken into account by using the same model function and the same value of particle moment but with a fixed relaxation time $\tau \gg 1 / \omega$ and allowing for a static field $B_{s t}$, which determines the previously defined order parameter. It accounts for the observed splitting of the intensities $I_{+}$and $I_{-}$in the time-independent contribution (see 

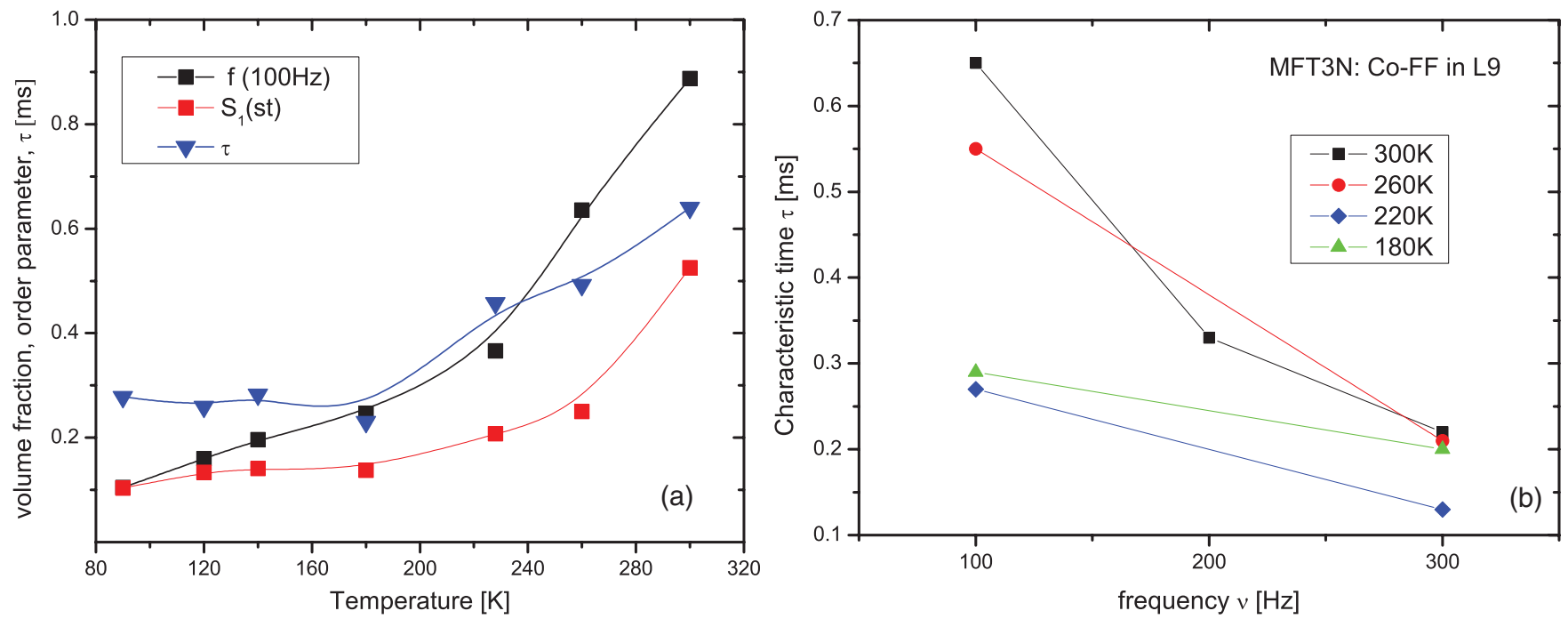

FIG. 7. (Color online) Model parameters for MFT3N as resulting from simultaneous fits of SANSPOL and POLARIS data in terms of the Debye-like relaxation model in Eq. (11)-(13). The decrease of $\tau$ and $f$ with decreasing temperature (a) as well as with increasing frequency (b) reflects the continuous freezing of particle moments as a function of the particle sizes.

Fig. 1). All POLARIS and SANSPOL data have simultaneously been fitted using theoretical values of the parameters $\mu$ (i.e., $\mu / k_{B} T$ increases with decreasing $T$ ) and $\Delta \eta_{\text {nuc }} / \Delta \eta_{\mathrm{mag}}$ of Table II, while the experimental phase shift $\phi_{0}$ and the structure factor $S(Q)$ in Eq. (10) have been adjusted as global parameters for both contributions. We note that no effect of phase shift $\phi_{2}$ has been observed in agreement with a value $S(Q)$ close to 1 for the analyzed ROI perpendicular to the field. The temperature and frequency variation of the volume fraction $f$ and characteristic time $\tau$ of mobile particles, as well as the order parameter $S_{1}^{\text {arr }}$ of the arrested particles, are shown in Fig. 7.

At a given temperature the volume fraction and the relaxation time $\tau$ decrease with increasing frequency. The model fit is rather sensitive to the parameter $\tau$, e.g., it was not possible to fit the data measured at $100 \mathrm{~K}$ and $300 \mathrm{~K}$ with the same $\tau$.

Thermal reorientation of the magnetic moment in a magnetic colloid can take place by Brownian rotation of the particle and/or by Néel relaxation of the particle moment. In the first mechanism the relaxation time $\tau_{B}$ depends on the hydrodynamic radius $R_{\mathrm{h}}$ and the viscosity $\eta$ of the solvent according to

$$
\tau_{B}=\frac{4 \cdot \pi \cdot \eta \cdot R_{h}^{3}}{k_{B} \cdot T}
$$

where $k_{B}$ is the Boltzmann constant and $T$ the absolute temperature. The Néel mechanism involves rotation of the magnetization vector inside a particle against an energy barrier $K_{a} \cdot V_{c}$ with a characteristic time $\tau_{N}$ given by

$$
\tau_{N}=\frac{1}{2 \cdot \pi \cdot f_{0}} \cdot \exp \left(\frac{K_{a} \cdot V_{c}}{k_{B} \cdot T}\right),
$$

where $f_{0}$ is a constant of the order of $10^{7} \mathrm{~s}^{-1}, K_{a}$ is a shape-dependent material constant, and $V_{c}$ is the volume of the magnetic core equal to $V_{c}=(4 / 3) \cdot \pi \cdot R_{c}^{3}$ for a sphere with radius $R_{c}$. The apparent characteristic time is given by $\tau_{\text {app }}^{-1}=\tau_{B}^{-1}+\tau_{N}^{-1}$, which means that the fastest mechanism dominates for individual particles.

For a single relaxation process one characteristic time $\tau$ should be observed at all frequencies, increasing in both cases with decreasing temperature. Here, we observe a slight decrease of $\tau$ with decreasing temperature (Fig. 7) and increasing frequency accompanied by a steep decrease of the fraction $f$ of mobile-particle moments around the freezing temperature of the solvent. This complex behavior is a clear signature for the dynamical nature of the local ordered domains as "living objects": The average time where an individual nanoparticle is attached to a larger ordered domain ("sticking time") must increase with decreasing temperature, leading to an increasing amount of larger aggregates. The Brownian relaxation time of these domains exceeds by far the period of the ac field and hence these domains appear as "static." Reversely, the fraction of particles and small aggregates which can keep up with the oscillating field becomes smaller, and their average apparent relaxation time will be shorter at lower temperature and higher frequencies. A more sophisticated model should take into account a (broad) distribution of relaxation times and particle moments related to the particle-size distribution. Nevertheless, the range of observed characteristic time between $600 \mu \mathrm{s}$ and $60 \mu \mathrm{s}$ at $100 \mathrm{~Hz}$ is of the same order of magnitude as relaxation times $\tau_{\mathrm{B}}(300 \mathrm{~K})=160 \mu$ s expected for Brownian rotational diffusion of individual core-shell particles of radius $R_{p}=R_{c}$ $+d_{s}=6.4 \mathrm{~nm}$ and a viscosity $\eta=0.2 \mathrm{~Pa}$ s for the liquid "L9," according to Eq. (14). In MFT3N the fast particle rotation causes the magnetic moments to align along the direction of the magnetic field. Similarly, a very broad range of relaxation times has been observed by SQUID relaxometry in iron-based nanoparticles, ${ }^{22}$ indicating a distribution of energy barriers despite the narrow range of particle sizes. Many features of this decay could be explained using a percolation model of particles with weak dipolar interactions. ${ }^{23}$

From polarized neutron data we can obtain clear information about the moment distribution of the static domains. When 

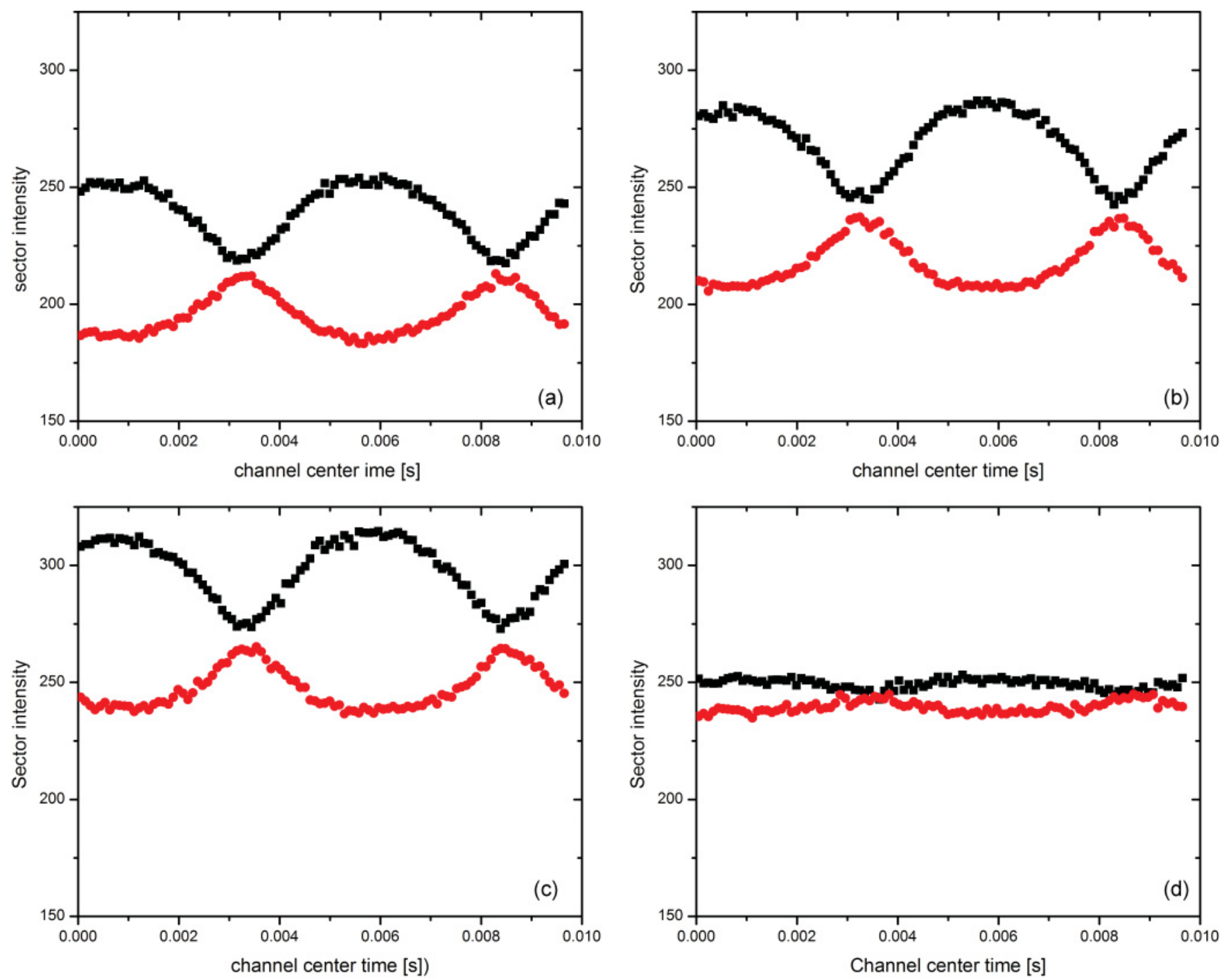

FIG. 8. (Color online) Time-dependent SANSPOL intensities of Co-FF 77-4 $I(Q)(+)_{\psi=90^{\circ}}$ and $I(Q)(-)_{\psi=90^{\circ}}$ at $300 \mathrm{~K}(\mathrm{a}), 240 \mathrm{~K}(\mathrm{~b})$, $150 \mathrm{~K}(\mathrm{~d})$, and $100 \mathrm{~K}(\mathrm{e})$. The symmetrical patterns indicate an isotropic moment distribution of the static particles.

$\tau$ is very large the intensity is damped to constant and isotropic values $I_{+}=I_{-}$as long as there is no static field. In a residual static field the intensities are different for $(+)$ and $(-)$ neutrons giving rise to the observed jump in the intensities at $B=0$ when the oscillating field changes its sign. At room temperature from the splitting of the intensities $I_{+}$and $I_{-}$an order parameter $S_{1}{ }^{\text {arr }}=0.6$ was derived, which was the same as measured in the static field and which corresponds to the magnetization according to the Langevin function in an external field of $0.02 \mathrm{~T}$. Almost all static moments are aligned along the preferred horizontal direction of the applied field. The number of particles attached to the static domains increases with decreasing temperature, while the order parameter decreases to $S_{1}{ }^{\text {arr }}=0.1$ ( $12 \%$ of the maximum value) in the frozen state at $100 \mathrm{~K}$, i.e., more and more static moments are distributed at random.

\section{B. Co-FF 77-4 (low viscosity)}

The SANSPOL intensities $I(Q)(+)$ and $I(Q)(-)$ averaged in sectors at $\psi=90^{\circ}$ with $\Delta \psi=30^{\circ}$, measured at $0.02 \mathrm{~T}$ and room temperature, can be described well by the form factors of noninteracting core-shell particles with the structural parameters reported in Table II. Since there is no significant interparticle correlation $(S(Q)=1)$ the intensities have been integrated in a $Q$ range between $0.2-0.6 \mathrm{~nm}^{-1}$, where only the dominant scattering contribution arises from the magnetic core. The time-dependent intensities for this ROI are shown in Fig. 8.

With decreasing temperature the absolute intensities and the amplitudes first increase and then decrease to nearly constant values below the solidification temperature around 120 K. Compared to the viscous sample MFT3N (Fig. 2) we observe rather symmetric patterns at all temperatures in the first- and second-half of the time period. The data have been first analyzed phenomenologically in terms of Eq. (7) with a contribution resulting from oscillating particle moments and a second one from static moments. The parameter $\mu / \mathrm{kT}$ was then found to increase when the temperature decreases until $150 \mathrm{~K}$ (which explains the increase of the absolute intensities) and then decreased rapidly in the solid state at $100 \mathrm{~K}$ while the fraction $f$ decreased continuously. 


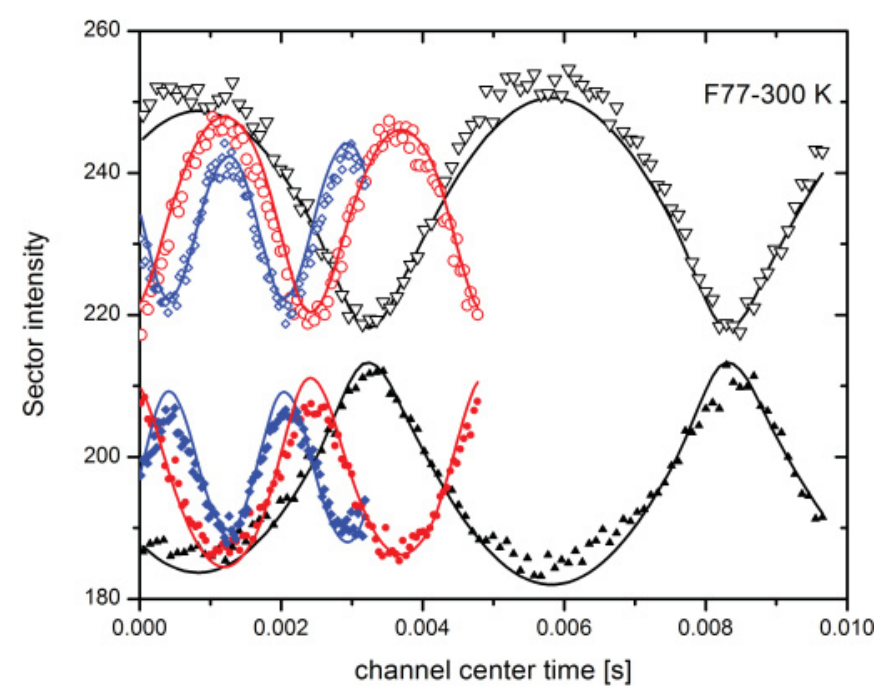

FIG. 9. (Color online) Co-FF 77-4 sample Co in D-toluene SANSPOL intensities $I(+)$ (solid symbols) and $I(-)$ (open symbols) measured at $300 \mathrm{~K}$ in an oscillating field of $0.02 \mathrm{~T}$ at $100 \mathrm{~Hz}$ (triangles), $200 \mathrm{~Hz}$ (circles), and $300 \mathrm{~Hz}$ (squares). Solid lines: fit to Debye-like relaxation model.

When the previously described Debye-like relaxation model was used the whole data set was consistently analyzed with a fraction $f$ for the mobile particles described by a relaxation time $\tau$ and a fraction $(1-f)$ of the static moments with $\tau \rightarrow \infty$. For latter, the order parameter $S_{1}{ }^{\text {arr }}$ has been determined from Eq. (13). At each temperature the data measured in an oscillating field of $0.02 \mathrm{~T}$ and at $v=100$, 200, and $300 \mathrm{~Hz}$ have been simultaneously fitted with the same value of $\tau$ together with the data measured in a static field of the same amplitude using the same global parameters of $\mu, S=1, F_{N} / F_{M}=-1.1$.

In the static field the order parameter determined at $300 \mathrm{~K}$ $S_{1}$ arr $(300 \mathrm{~K})=0.13$ corresponds exactly to the value expected for Langevin statistics in the applied field of 0.02 T. $S_{1}{ }^{\text {st }}$ decreases to $33 \%$ of this value for maximal moment alignment at $150 \mathrm{~K}$ and to only $20 \%$ in the solid state at $100 \mathrm{~K}$. In the oscillating field the simultaneous fit at three frequencies (Fig. 9) led to the temperature variation of $\tau$ and $f$ for the dynamic contribution, as shown in Fig. 10.

In the liquid state between $300 \mathrm{~K}$ and $150 \mathrm{~K}$ the relaxation time increases from about $4 \mu \mathrm{s}$ to $60 \mu \mathrm{s}$, which is nearly inversely proportional to $T$, as expected for Brownian relaxation $\tau=4 \pi \eta R^{3}{ }_{h} / 3 k_{\mathrm{B}} T$. From the slope $m=0.00166 \mathrm{~K} \mathrm{~s}$ of the linear fit of Fig. 10(b) and the hydrodynamic radius $R_{h}=$ $5 \mathrm{~nm}$ a viscosity of $\eta=144 \mathrm{mPas}$ is calculated, which is a reasonable value of for a mixture of $2 \%$ surfactants (oleic acide $\eta=3000 \mathrm{mPas})$ in toluene $(0.2 \mathrm{mPas})$.

Between 300 and $150 \mathrm{~K}$ the volume fraction of mobile particles decreases depending on the frequency (Fig. 10). In the solid state at $100 \mathrm{~K}$ only about $5 \%$ of the particle moments can follow the oscillating field. For the static particles the order parameter at all temperatures was found to be extremely low (around 0.0062) corresponding to only 4\% of the maximum value of moment alignment according to the Langevin function. This implies an almost random distribution of the frozen moments in the low viscous sample Co-FF77-4, contrary to the highly viscous sample MFT3N where the static moments were considerably aligned along the residual static-guide field.

\section{Magnetite-FF}

In a previous study ${ }^{16}$ we have shown that due to the actual value of the ratio $F_{N} / F_{M}=6.3$ (Table II) the SANS intensities in magnetite FF are highly sensitive to $S(Q)$. The structure factor was found to be time-dependent according to Eq. (10), which implied the presence of strong field-induced interparticle correlations resulting from the pronounced dipolar interactions between moments and leading to a distorted hexagonal arrangement of particles. ${ }^{13,14}$
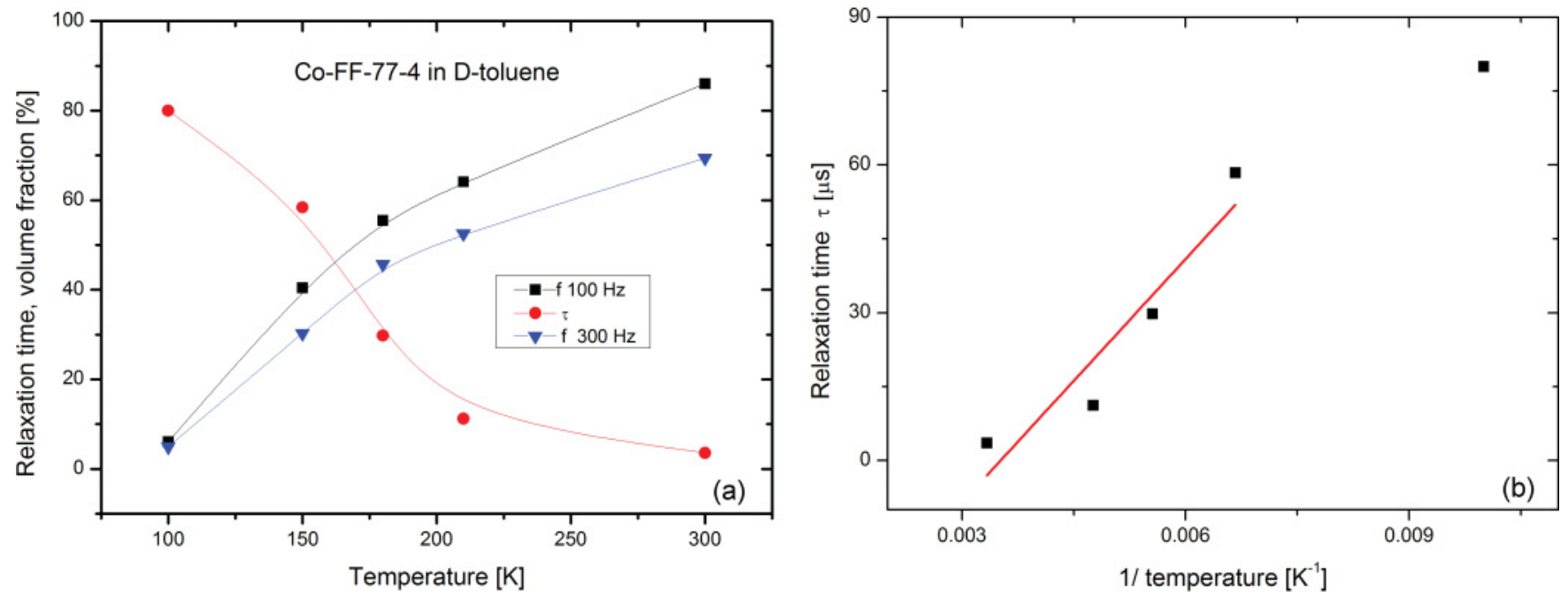

FIG. 10. (Color online) Co-FF77-4 of low viscosity. (a) Thermal variation of relaxation time $\tau$ (circles) and volume fraction of mobile particles measured at $100 \mathrm{~Hz}$ (squares) and $300 \mathrm{~Hz}$ (triangles) according to the Debye-like model (solid lines are guide for the eye). (b) The straight line represents a fit of the relaxation time $\tau$ at high temperatures according to the Brownian rotational diffusion assuming a temperature independent viscosity. 
TABLE III. Result of simultaneous fit of SANSPOL data of magnetite FF measured in an oscillating field of $B_{0}=20 \mathrm{mT}$ using the same theoretical values of $\mu_{0} / k_{\mathrm{B}} T=390$ (at $300 \mathrm{~K}$ ). Fit according to the phenomenological description and to the Debye-like relation model yielded either the phase shift $\phi_{1}$ or the relaxation time $\tau$.

\begin{tabular}{lcccccccc}
\hline \hline$v$ & $B_{0}$ & $B_{\mathrm{st}}$ & $f$ & $\phi_{0}$ & $\phi_{1}$ & $\phi_{2}$ & $S(Q)$ & $\tau$ \\
\hline $\mathrm{Hz}$ & $\mathrm{mT}$ & $\mathrm{mT}$ & $\%$ & $\circ$ & $\circ$ & $\circ$ & & $\mu \mathrm{s}$ \\
100 & 20 & $-1 \mathrm{e}-3$ & 99 & 9.2 & $12.2^{\circ}$ & 3.6 & 1.44 & 490 \\
200 & 20 & $-1 \mathrm{e}-3$ & 93 & -20.6 & 44.2 & 14.3 & 1.46 & 490 \\
300 & 20 & -0.02 & 52 & 22.3 & 43.5 & 56.0 & 1.23 & 490 \\
\hline \hline
\end{tabular}

Stroboscopic SANSPOL intensities $I(-)$ and $I(+)$ on magnetite measured at $300 \mathrm{~K}$ and shown in Fig. 11 have been fitted simultaneously (solid lines) with $L^{2}(x)$-dependent structure factors [Eq. (10)]. The results of fits according to the phenomenological description and to the Debye-like model are presented in Table III. In an ac-field of $100 \mathrm{~Hz}$ and an amplitude of $B_{0}=20 \mathrm{mT}$ almost all particles follow the oscillating field with a phase shift of $\varphi_{1}=13^{\circ}$ with respect to the applied field. At $300 \mathrm{~Hz} 93 \%$ of particle moments keep up with the oscillating field but with an increased phase $\phi_{1}=44.2^{\circ}$.

When a static field of $20 \mathrm{mT}$ is superimposed at $300 \mathrm{~Hz}$ we no longer expect a jump of the SANSPOL intensities from + to - since neutron spin and magnetic moment are parallel or antiparallel over the full period. Nevertheless the absolute intensities depend on the actual value of the delay between $M(t)$ and $B(t)$. In fact the patterns shown in Fig. 11 are well described by an oscillating contribution delayed by nearly the same value of $\phi_{1}=43.5^{\circ}$. The amount of arrested particle moments at this frequency is increased to $43 \%$, and the order parameter $S_{1}{ }^{\text {arr }}=0.3$ corresponds to maximum preferred orientation.

When the Debye-like relaxation model was used all curves could be simultaneously fitted with one relaxation time $\tau=$ $490 \mu \mathrm{s}$, which accounts for the observed phase shifts $\varphi_{1}$. It then turned out that an additional phase shift $\varphi_{2}$ must be taken into account. The observed values (Table III) correspond to a delay of the structure formation of $\delta t=\varphi_{2} / 2 \pi v=104 \mu \mathrm{s}$ at $100 \mathrm{~Hz}$ and $300 \mathrm{~Hz}$ and $B_{\mathrm{st}}=1 \mathrm{mT}$. In the static field of
$20 \mathrm{mT}$, where the coupling is more efficient, the set-up of the local dipolar ordering is delayed by $520 \mu \mathrm{s}$.

The example of magnetite FF shows impressively the sensitivity of the stroboscopic SANSPOL technique: The contribution of fully arrested particle moments aligned preferentially along the guide field jumps exactly when the magnetic field in the sample is zero, which precisely defines the experimental phase $\phi_{0}$. The strong field dependence of $S(Q$, $t$ ), according to Eq. (10), enables us to distinguish between the phase shift $\phi_{2}$ and $\phi_{1}$ arising from the delayed set-up of the local interparticle ordering and moment alignment in the oscillating field.

\section{SUMMARY}

The present study demonstrates that polarized neutrons can successfully be used in stroboscopic techniques where the response to a cyclic perturbation of the system by an oscillating field is measured as a time-dependent SANS signal. It has been shown that in the present frequency range the neutron spin follows the applied field adiabatically, and no depolarization occurs. Therefore the polarization-dependent scattering cross-sections could be evaluated for the applied oscillating magnetic field [Eq. (5) and Table I] and allowed simultaneous fits of the SANSPOL or POLARIS data as a "physical" contrast variation technique. The use of polarized neutrons not only reduces the ambiguity of data interpretation and enhances the reliability of the model parameters but also allows for additional information about the system not available with nonpolarized SANS. In the present case of the magnetic colloids an oscillating intensity was superimposed to a time-independent contribution in proportions variable with frequency and amplitude of the applied field and depending on temperature. The first contribution has been phenomenologically ascribed to a fraction of particle moments which can keep up with the oscillating field and the second one to the remaining part of "arrested" static moments. The use of polarized neutrons allows the model in three aspects to be refined:

First, the orientation distribution of the static moments is determined precisely by the jump of the constant SANSPOL intensity [e.g., from $I(+)$ to $I(-)$ ], which occurs when the
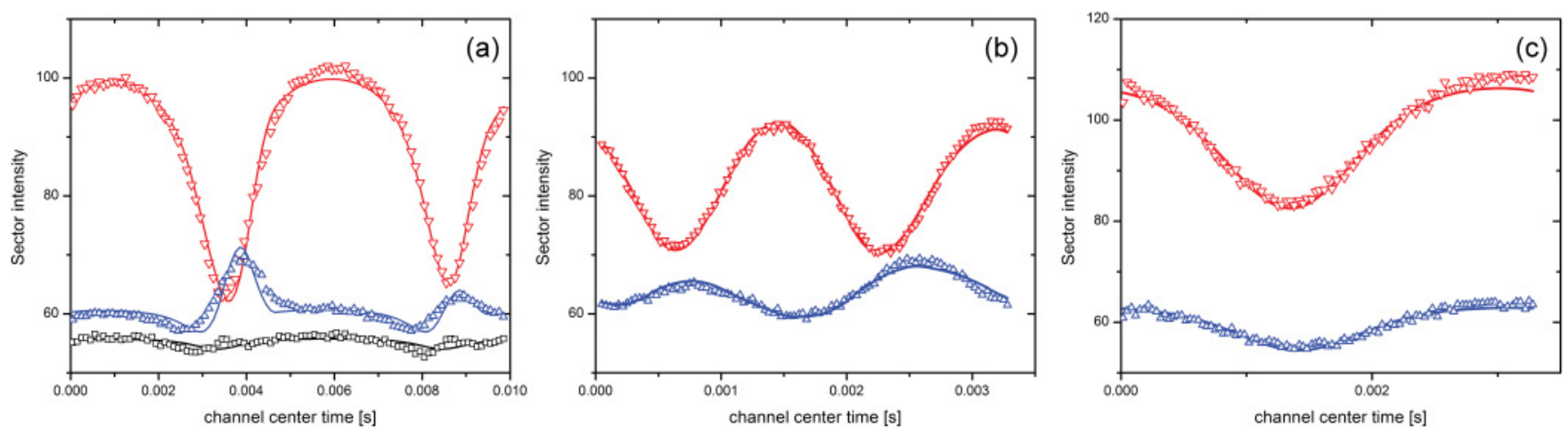

FIG. 11. (Color online) Time-dependent stroboscopic SANSPOL intensities of magnetite FF at $Q=0.3 \mathrm{~nm}^{-1}: I(-)(\mathrm{red} / \mathrm{down}$ triangles) and $I(+)$ (blue/up triangles) perpendicular and parallel (black squares) to an oscillating field of amplitude $B_{0}=20 \mathrm{mT}$ and frequency $100 \mathrm{~Hz}$ (a) or $300 \mathrm{~Hz}$ (b), (c). The static field $B_{\text {st }}$ was $0.1 \mathrm{mT}$ (a), (b) or $20 \mathrm{mT}$ (c). Solid lines are simultaneous fits including a delay between $B(t)$ and the magnetic response of $\phi_{1}=-18^{\circ}$ (a) and $-36^{\circ}$ (b), (c), respectively, and using $L^{2}(x)$ dependence of the structure factors. 
resulting magnetic field in the sample is zero and which defines the experimental phase $\phi_{0}$. The amount of alignment of the arrested moments along a preferred direction (of the guide field) has been quantified by an order parameter $S_{1}{ }^{\text {arr }}$. In MFT3 and magnetite $S_{1}$ arr corresponds to maximal alignment of the arrested moments along a preferred direction when the samples were in the liquid state and gradually decreases at lower temperatures when the solvent is frozen. In the low viscous sample Co-FF77-4 almost no preferred alignment was observed for the arrested fraction. This suggests that the local particle ordering in chains and hexagons induced by the much stronger dipolar coupling in the first two samples leads to a preferred moment orientation, which is conserved once the particles are arrested.

Second, for the fraction of mobile particles any delay of the moment relaxation with respect to the applied oscillating magnetic field leads to significant deformation of the SANSPOL and POLARIS intensities and allows a precise determination of the phase shift $\phi_{1}$. This frequency-dependent phase shift and damping of the intensity modulation have been explained quantitatively by a Debye-like relaxation model, leading to a characteristic relaxation time $\tau$ of the particles. The observed values of $\tau$ in the liquid state of all samples scale with the particle volume and viscosity which clearly confirms the Brownian relaxation mechanism. In the low viscous sample Co-FF77-4 the relaxation time increased with increasing reciprocal temperature, as expected. In the high viscous system MFT3N this effect is masked by a broad distribution of relaxation times. When the solvent starts to freeze out the viscosity increases rapidly, leading to extremely long relaxation times and hence to an increasing fraction of arrested moments. The residual relaxation dynamics observed in the frozen state might be governed by Brownian rotation of the core against the surfactant layer with finite viscosity or by Néel type relaxation of the smallest particles.

As a third aspect we mention the additional characteristic deformation of the SANSPOL and POLARIS intensities when strong interparticle interactions are relevant. This allows an additional phase shift $\phi_{2}$ to be extracted, which reflects the delayed set-up of the local interparticle ordering. In magnetite FF with the highest dipolar attraction energy, a clear delay in the formation of the distorted hexagonal particle arrangement was observed, which increases with frequency and magnitude of the inducing magnetic field $B(t)$.

The results will be compared to upcoming experiments using TISANE techniques where the characteristic relaxation times are directly measurable by the frequency variation of the oscillating field beyond $v>(2 \pi \tau)^{-1}$. *wiedenmann@ill.fr

${ }^{1}$ A. Wiedenmann, U. Keiderling, R. May, and C. Dewhurst, Physica B 385, 453 (2006).

${ }^{2}$ A. Wiedenmann, U. Keiderling, K. Habicht, M. Russina, and R. Gähler, Phys. Rev. Lett. 97, 057202 (2006).

${ }^{3}$ A. Wiedenmann, U. Keiderling, M. Meissner, D. Wallacher, R. Gähler, R. P. May, S. Prévost, M. Klokkenburg, B. Erné, and J. Kohlbrecher, Phys. Rev. B 77, 184417 (2008).

${ }^{4}$ D. E. Madsen, M. T. Hansen, and S. Mørup, J. Phys. Condens. Matter 20, 345209 (2008).

${ }^{5}$ R. Kotitz, W. Weitschies, L. Trahms, and W. Semmler, J. Magn. Magn. Mater. 201, 102 (1999).

${ }^{6}$ H. Casalta, P. Schleger,C. Bellouard, M. Hennion, I. Mirebeau, G. Ehlers, B. Farago, J. L. Dormann, M. Kelsch, M. Linde, and F. Phillipp, Phys. Rev. Lett. 82, 1301 (1999).

${ }^{7}$ F. Gazeau, F. Boué, E. Dubois, and R. Perzynski, J. Phys. Condens. Matter 15, S1305 (2003).

${ }^{8}$ M. F. Hansen, F. Bodker, S. Morup, K. Lefmann, K. N. Clausen, and P. A. Lindgard, Phys. Rev. Lett. 79, 4910 (1997).

${ }^{9}$ J. Lal, D. Abernathy, L. Auvray, O. Diat, and G. Grübel, Eur. Phys. E 4, 263 (2001).

${ }^{10}$ J. C. Bacri, A. Cebers, A. Bourdon, G. Demouchy, B. M. Heegaard, and R. Perzynski, Phys. Rev. Lett. 74, 5032 (1995).

${ }^{11}$ S. Behrens, H. Bönnemann, H. Modrow, V. Kempter, W. Riehemann, A. Wiedenmann, S. Odenbach, S. Will, L. Trahms, R. Hergt, R. Müller, K. Landfester, A. Schmidt, D. Schüler, and R. Hempelmann, In: Colloidal Magnetic Fluids, edited by
S. Odenbach, Lecture Notes in Physics 763 (Springer, Berlin, Heidelberg, 2009), pp. 1-82, DOI 10.1007/978-3-540-85382-9.

${ }^{12}$ A. Wiedenmann, A. Hoell, M. Kammel, and P. Boesecke, Phys. Rev. E 68, 031203 (2003).

${ }^{13}$ M. Klokkenburg, B. H. Erné, J. D. Meeldijk, A. Wiedenmann, A. V. Petukhov, R. P. A. Dullens, and A. P. Philipse, Phys. Rev. Lett. 97, 185702 (2006).

${ }^{14}$ M. Klokkenburg, B. H. Erné, A. Wiedenmann, A. V. Petukhov, and A. P. Philipse, Phys. Rev. E 75, 051408 (2007).

${ }^{15}$ R. Pynn, J. B. Hayter, and S. W. Charles, Phys. Rev. Lett. 51, 710 (1983).

${ }^{16}$ J. Kohlbrecher, A. Wiedenmann, and H. Wollenberger, Z. Physik B 104, 1 (1997).

${ }^{17}$ A. Wiedenmann, Physica B 356, 246 (2005).

${ }^{18}$ D. Honecker, A. Ferdinand, F. Döbrich, C. D. Dewhurst, A. Wiedenmann, C. Gomez-Polo, K. Suzuki, and A. Michels, Eur. Phys. J. B 76, 209 (2010).

${ }^{19}$ A. K. Petoukov, V. Guillard, K. H. Andersen, E. Bourgeat-Lami, R. Chung, H. Humblot, D. Jullien, E. Lelievre-Berna, T. Soldner, F. Tasset, and M. Thomas, Nuclear Instr. and Methods B, 560, 480 (2006).

${ }^{20}$ U. Keiderling, A. Wiedenmann, A. Rupp, J. Klenke, and W. Heil, Meas. Sci. Technol. 14, 034009 (2008).

${ }^{21}$ J. Kohlbrecher, SASFIT package, [http://kur.web.psi.ch/sans1/ SANSSoft/sasfit.html].

${ }^{22}$ R. V. Chamberlin, K. D. Humfeld, D. Farrell, S. Yamamuro, Y. Ijiri, and S. A. Majetich, J. Appl. Phys. 91, 6961 (2002).

${ }^{23}$ R. V. Chamberlin and M. R. Scheinfein, Science 260, 1098 (1993). 